\title{
Dispositivo de bajo costo para disminuir el efecto de aerosol durante la endoscopia de vías digestivas altas en COVID-19
}

\section{Low cost devices to reduce aerosol effect during upper gastrointestinal endoscopy in COVID-19}

Ricardo Villarreal, ${ }^{1}$ Luis Felipe Cabrera, ${ }^{2}$ Mauricio Pedraza,,${ }^{3}$ Jhon Eric Cuervo, ${ }^{4}$ Jorge Hernán Santos, ${ }^{5}$ José Roberto Jurado. ${ }^{6}$

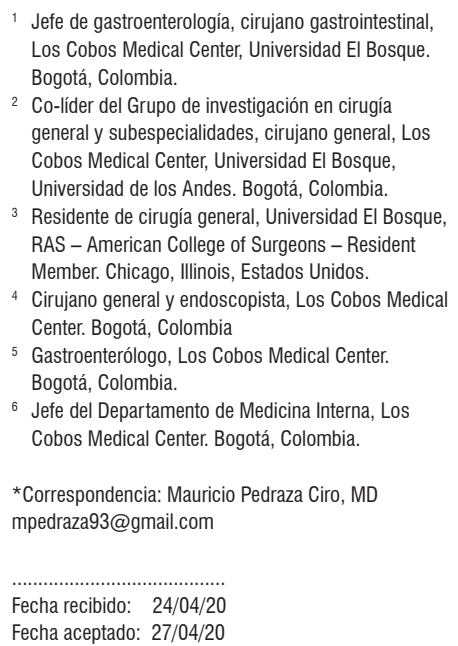

2 Co-líder del Grupo de investigación en cirugía general y subespecialidades, cirujano general, Los Cobos Medical Center, Universidad El Bosque, Universidad de los Andes. Bogotá, Colombia.

${ }^{3}$ Residente de cirugía general, Universidad El Bosque, RAS - American College of Surgeons - Resident

Member. Chicago, Illinois, Estados Unidos.

${ }^{4}$ Cirujano general y endoscopista, Los Cobos Medical Center. Bogotá, Colombia

5 Gastroenterólogo, Los Cobos Medical Center. Bogotá, Colombia.

6 Jefe del Departamento de Medicina Interna, Los Cobos Medical Center. Bogotá, Colombia.

*Correspondencia: Mauricio Pedraza Ciro, MD mpedraza93@gmail.com

Fecha recibido: $24 / 04 / 20$

Fecha aceptado: $27 / 04 / 20$

\begin{abstract}
Resumen
Los procedimientos endoscópicos, al estar asociados a la exposición de la vía aérea superior, tienen un mayor riesgo de transmisión del virus SARS-CoV-2; por esta razón, es necesario el planteamiento de intervenciones para atenuar o eliminar la exposición a éste. En este artículo nos permitimos presentar una estrategia de mitigación de bajo costo, ya que con el progreso de la pandemia se podría estar en un estado de austeridad de recursos económicos, lo cual haría estos dispositivos totalmente válidos.
\end{abstract}

\section{Palabras clave}

COVID-19, efecto, aerosol y endoscopia.

\begin{abstract}
Keywords

COVID-19, effect, aerosol and endoscopy.
\end{abstract}

Endoscopic procedures, as they are associated with exposure of the upper airway, have a higher risk of transmission of the SARS-CoV-2 virus; so, it is necessary to consider interventions to mitigate or eliminate exposure to it. In this article, we allow ourselves to present a low-cost mitigation strategy, since with the progress of the pandemic it could be in a state of austerity of economic resources, making these devices totally valid.

\section{INTRODUCCIÓN}

Los coronavirus son una familia de virus que causan enfermedades en personas, y que también circulan entre los animales. La cepa COVID-19, causante del brote actual, se identificó por primera vez en Wuhan, en la provincia china de Hubei, China, en diciembre de 2019. Meses después, la Organización Mundial de la Salud (OMS) lo elevó a la categoría de pandemia y decretó emergencia sanitaria, ahora con más de 2585468 casos y 178481 muertes a nivel mundial. En Colombia, a la fecha, según reportes del Ministerio de Salud, tenemos 4149 casos y 196 muertes. Esta infección se presenta en forma de fiebre, tos seca y disnea leve en la mayoría de los casos, si bien puede evolucionar hacia neumonía grave y un eventual síndrome de dificultad respiratoria del adulto, similar al síndrome respiratorio agudo grave (SARS, por sus siglas en inglés) descrito en 2003 y al síndrome respiratoria de Medio Oriente (MERS, por sus siglas en inglés) de 2012. El SARS-CoV-2 (COVID-19) es un 
patógeno respiratorio tipo $\mathrm{ARN}$, el cual se ha detectado principalmente en muestras del tracto respiratorio superior e inferior. Los viriones COVID-19 tienen un tamaño de aproximadamente 0,125 micras, y se transmiten más comúnmente como gotas de agua respiratoria más grandes (> 20 micras) (1-5).

\section{MATERIALES Y MÉTODOS}

Presentamos la aplicación de un dispositivo de bajo costo desechable (mascarilla convencional con modificación de una incisión de 1 a 1,5 cm) (Figuras 1 y 2) cuya función es disminuir la difuminación de los aerosoles generados durante una endoscopia de vías digestivas altas (Figura 3), y así mitigar el riesgo de transmisión e infección del SARS$\mathrm{CoV}-2$ para los profesionales de la salud que se encuentren en la sala de endoscopia, quienes se encuentran a menos de 1 metro del paciente y están en riesgo permanente.

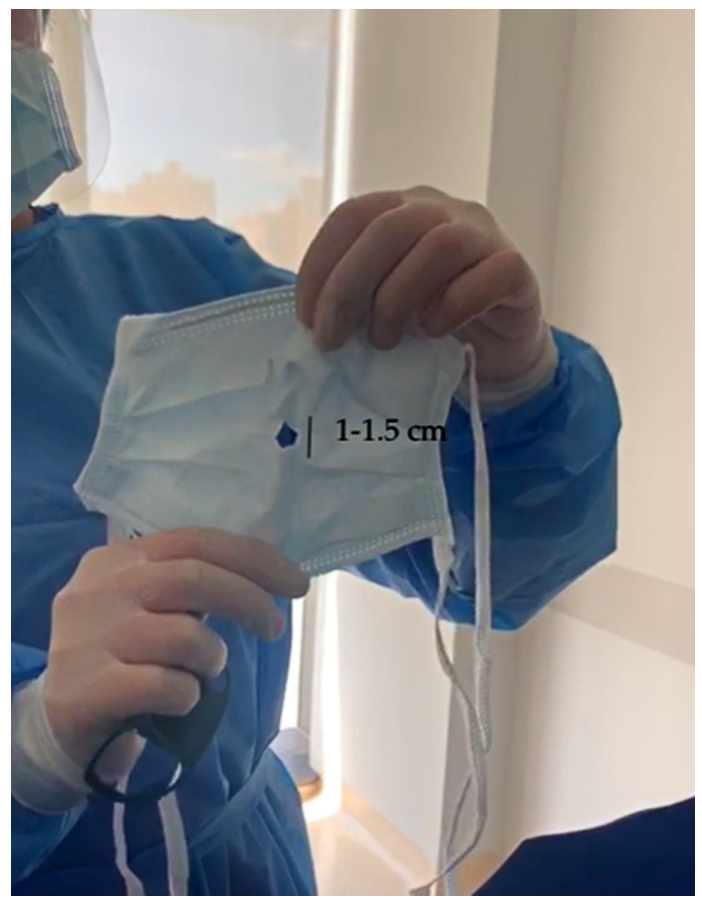

Figura 1. Mascarilla convencional con modificación. Fuente: imágenes propias de los autores.

El uso de este dispositivo no alteró el desarrollo de ninguno de los procedimientos endoscópicos. Se le explicaba al paciente de forma clara sobre el beneficio de la protección que brindaba este dispositivo, tanto para él como para el personal médico tratante. Además, el médico gastroenterólogo y los ayudantes utilizaron todos los elementos de protección para limitar el contagio por gotas de este virus (Figura 4).

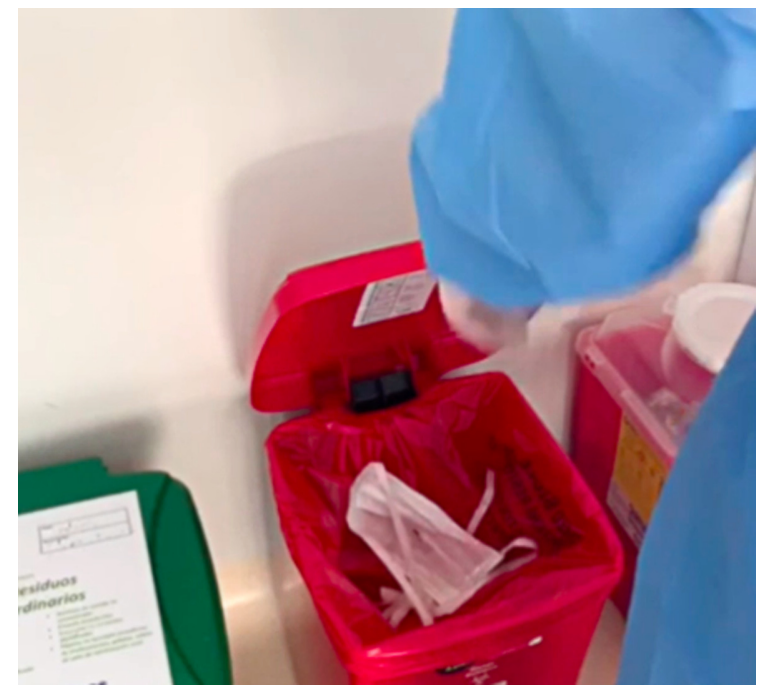

Figura 2. Dispositivo de bajo costo desechable. Fuente: imágenes propias de los autores.

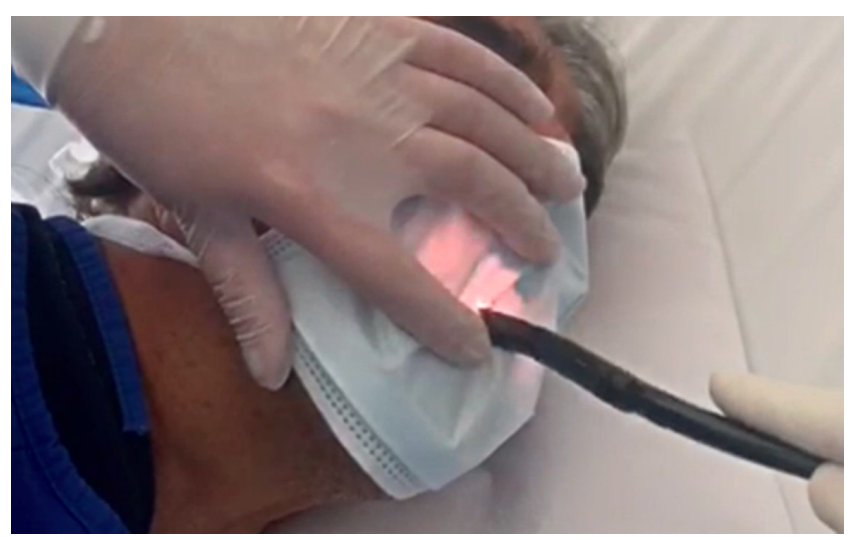

Figura 3. Procedimiento endoscópico. Fuente: imágenes propias de los autores.

\section{DISCUSIÓN Y CONCLUSIÓN}

William F. Wells describió en 1930 la transmisión de enfermedades respiratorias, como la tuberculosis, por medio de las emisiones de gotas respiratorias dicotomizadas en macro y microgotas. Los aerosoles son partículas menores de $5 \mu \mathrm{m}$ de diámetro que se producen cuando una corriente de aire se mueve a través de una superficie con película de líquido, su tamaño es inversamente proporcional a la velocidad del aire, y pueden permanecer en el ambiente por largos períodos de tiempo ( 1 a 2 horas) y recorrer distancias mayores a 1 metro. Un procedimiento generador de aerosol es definido como cualquier procedimiento médico que pueda inducir la producción de aerosoles de cualquier tamaño, incluyendo los núcleos de gotas (6-10). 


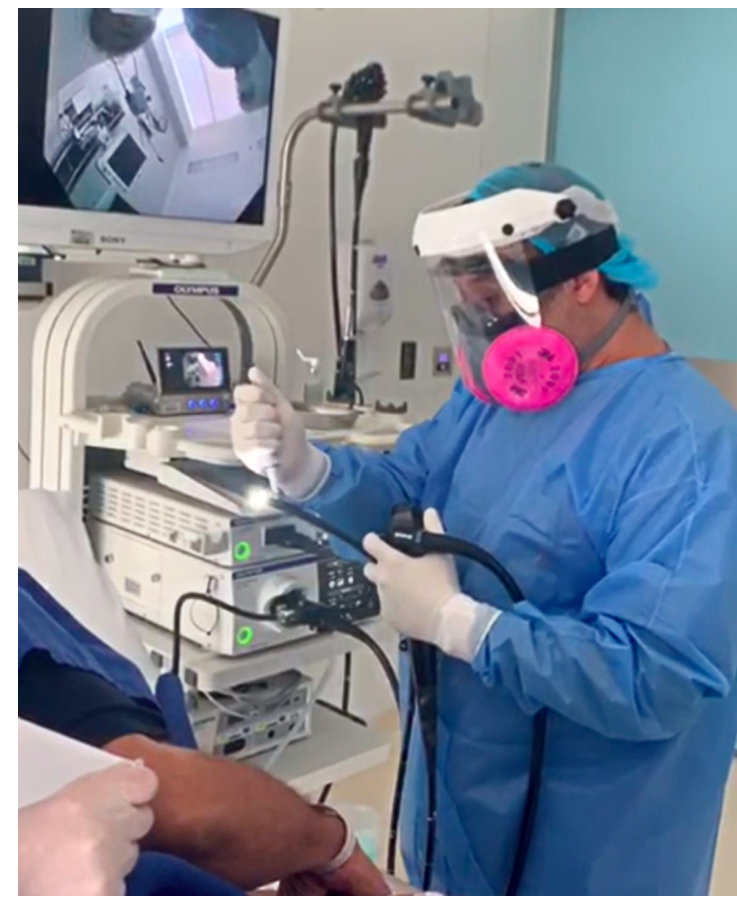

Figura 4. Elementos de protección para limitar el contagio por gotas de este virus. Fuente: imágenes propias de los autores.

El virus también puede ser aerosolizado y transmitido en microgotas ( $<10$ micras) en suspensión de gas. El tamaño de las partículas tiene implicaciones para el tiempo de suspensión y los requisitos de filtración. El ARN del SARS-CoV-2 también se ha detectado en muestras de sangre y heces, pero se desconoce si el virus está presente en estas muestras extrapulmonares. La alta tasa de contagio y la patogénesis de la enfermedad dependen del anclaje del virus a las células humanas a través del dominio de la unión a receptores de la glucoproteína transmembrana $\mathrm{S}$, el reconocimiento de receptores celulares como la enzima convertidora de angiotensina 2 (ECA2) y el ingreso al citoplasma mediante una serina proteasa transmembrana (TMPRSS) (1-6).

Además, aunque se puede detectar ARN viral en la sangre, no se ha documentado ninguna transmisión de SARSCoV-2 a través de esta ruta. En retrospectiva, lo que hemos podido aprender de otras epidemias virales es que el riesgo de transmisión de enfermedades virales en endoscopia y laparoscopia es prácticamente nulo. Aunque COVID-19 es una enfermedad nueva, virus respiratorios similares, como la gripe y otros coronavirus (SARS y MERS-CoV), no han demostrado evidencia de transmisión de la enfermedad a través del gas del endoscopio, el neumoperitoneo ni del humo quirúrgico (6-12).

Por otro lado, el $\mathrm{ADN}$ viral de los patógenos transmitidos por la sangre, como la hepatitis B y el virus de la inmunode- ficiencia humana (VIH), se ha detectado en el humo quirúrgico después del uso de energía durante la cirugía (por ejemplo, electrocirugía); sin embargo, no hay evidencia que indique que el uso de electrocirugía durante la endoscopia o la laparoscopia aumente el riesgo de transmisión de la enfermedad a través del gas del endoscopio, el humo quirúrgico o el neumoperitoneo $(5,6)$. Además, la cirugía en pacientes con VIH y hepatitis $\mathrm{B}$ y $\mathrm{C}$ ha estado en curso durante décadas, $\mathrm{y}$ no se ha documentado un mayor riesgo de transmisión por el uso del neumoperitoneo para los cirujanos, anestesiólogos o el resto del personal en el quirófano, lo cual se puede extrapolar a la endoscopia (6-13).

Los procedimientos endoscópicos son generadores de aerosoles, ya sean aerosoles provenientes del paciente o del endoscopio, que se pueden producir por la respuesta del paciente al estímulo del endoscopio durante su introducción, por flatos y descargas de líquidos como producto de la preparación del colon y por la manipulación de instrumentos por los canales de trabajo del endoscopio para la toma de muestras y drenaje de fluidos (14-16). El gas generado contiene aerosoles que pueden transportar el virus en pacientes que dieron positivo para COVID-19, puede ocasionar la contaminación del aire y de las superficies y, de esta manera, la transmisión del virus (17-19).

Para concluir, las herramientas para minimizar la exposición a aerosoles son indispensable en la era de COVID-19; sin embargo, la posibilidad de entrar en la fase 1 llama a los clínicos a buscar diferentes formas económicas y eficaces que mitiguen la exposición.

\section{REFERENCIAS}

1. Zheng $\mathrm{MH}$, Boni L, Fingerhut A. Minimally Invasive Surgery and the Novel Coronavirus Outbreak: Lessons Learned in China and Italy. Ann Surg. 2020 Mar 26. https: / / doi.org/10.1097/SLA.0000000000003924

2. Kwak HD, Kim SH, Seo YS, Song KJ. Detecting hepatitis B virus in surgical smoke emitted during laparoscopic surgery. Occup Environ Med. 2016 Dec;73(12):857-863. https:// doi.org/10.1136/oemed-2016-103724

3. van Doremalen N, Bushmaker T, Morris DH, Holbrook MG, Gamble A, Williamson BN, et al. Aerosol and Surface Stability of SARS-CoV-2 as Compared with SARS-CoV-1. N Engl J Med. 2020;382(16):1564-1567. https://doi. org/10.1056/NEJMc2004973

4. Baron S, Fons M, Albrecht T. Viral Pathogenesis. En: Baron S, editor. Medical Microbiology. 4. ${ }^{\text {a }}$ edición. Galveston (TX): University of Texas Medical Branch at Galveston; 1996.

5. Kim JM, Chung YS, Jo HJ, Lee NJ, Kim MS, Woo SH, et al. Identification of Coronavirus Isolated from a Patient in Korea with COVID-19. Osong Public Health Res Perspect. 2020;11(1):3-7. https://doi.org/10.24171/j. phrp.2020.11.1.02 
6. Johnston ER, Habib-Bein N, Dueker JM, Quiroz B, Corsaro E, Ambrogio M, et al. Risk of bacterial exposure to the endoscopist's face during endoscopy. Gastrointest Endosc. 2019;89(4):818-824. https://doi.org/10.1016/j. gie.2018.10.034

7. Soetikno R, Teoh AY, Kaltenbach T, Lau JY, Asokkumar R, Cabral-Prodigalidad $\mathrm{P}$, et al. Considerations in performing endoscopy during the COVID-19 pandemic. Gastrointest Endosc. 2020 Mar 27. pii: S0016-5107(20)34033-5. https://doi.org/10.1016/j.gie.2020.03.3758

8. Zhang Y, Zhang X, Liu L, Wang H, Zhao Q. Suggestions for infection prevention and control in digestive endoscopy during current 2019-nCoV pneumonia outbreak in Wuhan, Hubei province, China. Endoscopy. 2020;52(4):312-314. https://doi.org/10.1055/a-1128-4313

9. Morris SN, Fader AN, Milad MP, Dionisi HJ. Understanding the "Scope" of the Problem: Why Laparoscopy is Considered Safe During the COVID-19 Pandemic. J Minim Invasive Gynecol. 2020 Apr 2. pii: S1553-4650(20)301710. https://doi.org/10.1016/j.jmig.2020.04.002

10. Repici A, Maselli R, Colombo M, Gabbiadini R, Spadaccini $\mathrm{M}$, Anderloni A, et al. Coronavirus (COVID-19) outbreak: what the department of endoscopy should know. Gastrointest Endosc. 2020 Mar 14. pii: S0016-5107(20)30245-5. https://doi.org/10.1016/j.gie.2020.03.019.

11. Ong SWX, Tan YK, Chia PY, Lee TH, Ng OT, Wong MSY, et al. Air, Surface Environmental, and Personal Protective Equipment Contamination by Severe Acute Respiratory Syndrome Coronavirus 2 (SARS-CoV-2) From a Symptomatic Patient. JAMA. 2020 Mar 4. https://doi. org/10.1001/jama.2020.3227

12. Balibrea JM, Badia JM, Rubio I, Martín E, Álvarez E, García $S$, et al. Manejo quirúrgico de pacientes con infección por COVID-19. Recomendaciones de la Asociación Española de Cirujanos. Cir Esp. 2020. https://doi.org/10.1016/j. ciresp.2020.03.001
13. Snyman L, Makulana T, Makin JD. A randomised trial comparing laparoscopy with laparotomy in the management of women with ruptured ectopic pregnancy. S Afr Med J. 2017;107(3):258-263. https://doi.org/10.7196/ SAMJ.2017.v107i3.11447

14. Mintz Y, Arezzo A, Boni L, Chand M, Brodie R, Fingerhut A. A Low Cost, Safe and Effective Method for Smoke Evacuation in Laparoscopic Surgery for Suspected Coronavirus Patients. Annals of Surgery. 2020. https://doi. org/10.1097/SLA.0000000000003965

15. Vigneswaran Y, Prachand VN, Posner MC, Matthews JB, Hussain M. What Is the Appropriate Use of Laparoscopy over Open Procedures in the Current COVID-19 Climate? J Gastrointest Surg. 2020 Apr 13. https://doi.org/10.1007/ s11605-020-04592-9.

16. American College of Surgeons [Internet]. The American College of Surgeons ACS COVID-19 and Surgery [24 de marzo de 2020] Disponible en: https://www.facs.org/ covid-19/clinical-guidance

17. Society of American Gastrointestinal and Endoscopic Surgeons [Internet]. The Society of American Gastrointestinal and Endoscopic Surgeons SAGES Recommendations Regarding Surgical Response to COVID-19 Crisis 2020. Disponible en: https: / /www.sages. org/recommendations-surgical-response-covid-19/

18. Royal College of Surgeons of England [Internet]. The Royal College of Surgeons Updated Intercollegiate General Surgery Guidance on COVID-19. 2020. Disponible en: https://www.rcseng.ac.uk/coronavirus/joint-guidance-forsurgeons-v2

19. Otero W, Gómez M, Angel L, et al. Basic considerations regarding endoscopic procedures during the COVID19 pandemic. Rev Colomb Gastroenterol. 2020;35(1). https://doi.org/10.22516/25007440.526 